\title{
Теоретический анализ качества передачи сигнала в волоконно-оптических линиях связи
}

\author{
А.С. Скидин ${ }^{*}$ O.С. Сидельников, М.П. Федорук \\ Новосибирский государственный университет \\ *E-mail: ask@skidin.org
}

DOI: $10.31868 /$ RFL2020.179-180

В современных волоконно-оптических линиях связи (ВОЛС) с целью преодоления ограничений, накладываемых нелинейностью в волокне, используется множество специализированных методов обработки сигнала [1], таких как обратное распространение, адаптивная модуляция, машинное обучение. Оптимизация данных техник требует простых методов оценки качества выходного сигнала. В ходе исследования нами был предложен метод оценки качества нелинейной ВОЛС с использованием только начального сигнала. Для верификации результатов был использован сигнал на основе 16-QAM-OFDM с 1024 подканалами.

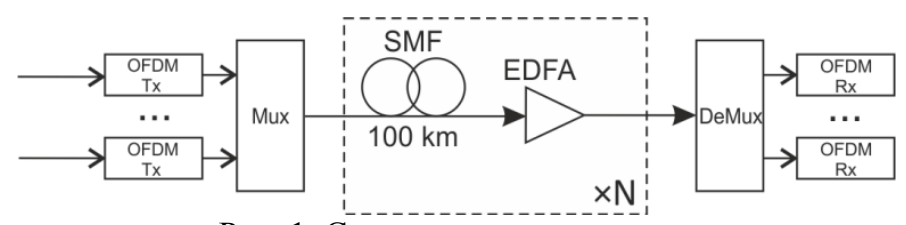

Рис. 1. Схема передачи сигнала.

Передача сигнала по волокну в схеме 1 описывается широко известным нелинейным уравнением Шредингера:

$$
\frac{\partial A}{\partial z}=-\frac{\alpha}{2} A-i \frac{\beta_{2}}{2} \frac{\partial^{2} A}{\partial t^{2}}+i \gamma|A|^{2} A .
$$

Здесь $A(z, t)$ - медленно меняющаяся огибающая сигнала, потери в волокне $\alpha=0.2$ дБ/км, хроматическая дисперсия $\beta_{2}=25$ пс $^{2} /$ км, коэффициент нелинейности $\gamma=1.4$ 1/Вт/км. Введем следующие обозначения: $x=z \cdot \alpha / 2, \quad \tau=t \sqrt{\alpha / \beta_{2}}$, $u(x, \tau)=A(z, t) \cdot \alpha / 2$. Тогда можно преобразовать уравнение (1) в следующую форму:

$$
\frac{\partial u}{\partial x}=-u-i \frac{\partial^{2} u}{\partial \tau^{2}}+i|u|^{2} u K
$$

где $\quad K=8 \gamma / \alpha^{3}$. Если $u(x, \tau)=\sqrt{p(x, \tau)} \cdot \exp (i \rho(x, \tau)), \quad$ можно $\quad$ записать следующие уравнения на эволюцию фазы и мощности:

$$
\frac{\partial p}{\partial x}=-2 p+2 \frac{\partial}{\partial \tau}\left(p \cdot \frac{\partial \rho}{\partial \tau}\right), \frac{\partial \rho}{\partial x}=\frac{1}{4 p^{2}}\left(\frac{\partial p}{\partial \tau}\right)^{2}-\frac{1}{2 p} \frac{\partial^{2} p}{\partial \tau^{2}}+\left(\frac{\partial \rho}{\partial \tau}\right)^{2}+p K
$$

Рассмотрим начальную функцию $u(x=0 ; t)$. Будем предполагать, что $u(x=0 ; t)$ соответствует OFDM-сигналу с М подканалами (М принимает значения порядка нескольких сотен). Также будем предполагать, что мощность сигнала достаточно велика, чтобы передача сигнала была нелинейной. При этих допущениях рассмотрим мгновенные значения члена $\frac{\partial}{\partial \tau}\left(p(x=0, \tau) \cdot \frac{\partial \rho(x=0, \tau)}{\partial \tau}\right)$ как случайную переменную $\xi$.

Из системы (3) можно видеть, что эволюция мощности сигнала ( $\partial p / \partial x)$ зависит от стандартных потерь сигнала (детерминированная часть) и от $x$ (стохастическая часть). С целью улучшения качества сигнала необходимо 
минимизировать влияние на сигнал члена $\xi=\frac{\partial}{\partial \tau}\left(p \cdot \frac{\partial \rho)}{\partial \tau}\right)$. С точки зрения статистики можно показать, что, если число OFDM-подканалов в начальном сигнале большое, то $E\left[\partial^{n} \rho / \partial \tau^{n}\right]=0$ для любого $n>0$ (E[x] - математическое ожидание случайной величины х). Тогда $E[\xi]=0$. Обозначим за $\sigma_{\xi}=\sqrt{E\left[\xi^{2}\right]}$ среднеквадратичное отклонение $\xi$. Можно видеть, что $\sigma_{\xi}$ пропорционально мощности сигнала. Также $\sigma_{\xi}$ зависит от пиков мощности, поскольку в пиковых точках производные сигнала велики. При оптимизации передачи сигнала (например, при адаптивной модуляции 256-QАМ и 1024-QАМ сигналов) $\sigma_{\xi}$ можно рассматривать как индикатор, способный усовершенствовать процесс оптимизации: достаточно только построить начальные сигналы и сравнить их по величине $\sigma_{\xi}$ с целью определения, какой из них имеет минимальную $\sigma_{\xi}$.

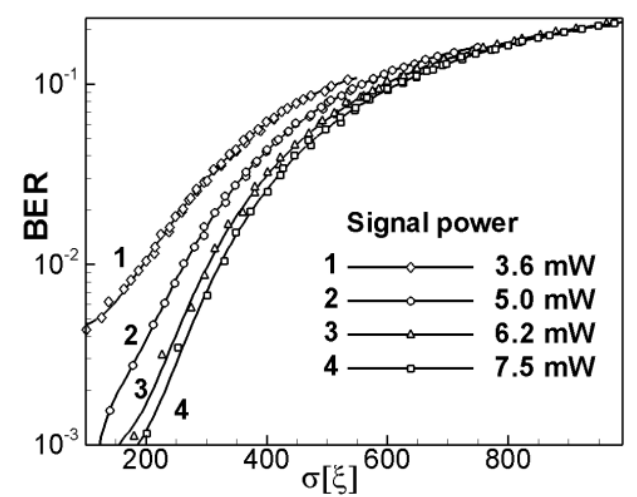

Рис. 2. Зависимость BER от среднеквадратического отклонения $\xi$.

Были проведены численные расчеты передачи 16-QAM-OFDM сигнала с 1024 подканалами по линии, состоящей из 10 периодических секций (рис. 1) длиной 100 км каждая. На рис. 2 показана зависимость частоты битовых ошибок после 1000 км от $\sigma_{\xi}$ для различных начальных сигналов. Можно легко заметить, что BER растет экспоненциально с ростом $\sigma_{\xi}$. Для оптимизации свойств ВОЛС при помощи адаптивной модуляции [3] и предложенного в ходе данного исследования метода достаточно получить начальный сигнал при помощи одного из методов адаптивной модуляции, а затем оценить $\sqrt{E\left[\xi^{2}\right]}$ для наборов вероятностей, дающих желаемую избыточность выходного кода, и выбрать набор с наименьшим $\sqrt{E\left[\xi^{2}\right]}$.

Работа Скидина А.С. (теоретический анализ) была выполнена при поддержке проекта РФФИ №18-31-20027. Работа Сидельникова О.С. была поддержана государственным заданием на проведение фундаментальных исследований №FSUS2020-0034.

\section{Литература}

[1] J.C.Cartledge, F.P.Guiomar, et al. Opt. Express 25, 1916-1936 (2017).

[2] A. Skidin, O. S. Sidelnikov, et al. Opt. Express 24, 30296-30308 (2016).

[3] J.Cho, P.J. Winzer, J. Lightwave Technol. 37, 1590-1607 (2019). 